\title{
NUEVA LECTURA DE LA INSCRIPCIÓN IBÉRICA SOBRE PIEDRA CONSERVADA EN EL MUSEO DE CRUZY (HÉRAULT)
}

\section{A NEW READING OF THE IBERIAN INSCRIPTION ON STONE PRESERVED IN THE MUSEUM OF CRUZY (HÉRAULT)}

\author{
Noemí Moncunill, Joan Ferrer i Jane y Joaquín Gorrochategui \\ Université de Paris Sorbonne - Grupo Littera, UB - Instituto de Ciencias de la Antigüedad, UPVIEHU \\ nmoncunill@gmail.com \\ joan.ferrer.i.jane@gmail.com \\ joaquin.gorrochategui@ehu.eus \\ ORCID: 0000-0001-5433-2156
}

DOI: $10.1387 /$ veleia.16981

Resumen: Presentamos una nueva edición de la inscripción ibérica sobre piedra hallada en los alrededores de Ensérune y conservada en el Museo de Cruzy (Hérault, Francia). La nueva propuesta de lectura, resultado de autopsia de la pieza, obliga a reconsiderar el análisis de la onomástica contenida en el texto, abriendo varias vías de interpretación.

Palabras clave: epigrafía ibérica, lengua ibérica, Ensérune, onomástica.

Abstract: This article presents a new edition of the Iberian inscription on stone found near Ensérune and preserved in the Musée de Cruzy (Hérault, France). The new reading proposal, based on the autopsy of the inscription, makes it necessary to reconsider the onomastics attested in the text, and opens new possibilities for its interpretation.

Keywords: Iberian epigraphy, Iberian language, Ensérune, onomastics.

Recibido: 13-04-2016

Informado: 16-05-2016

Definitivo: 27-05-2016

\section{INTRODUCCIÓN ${ }^{1}$}

Con el nombre de "piedra de Cruzy» se conoce una de las inscripciones ibéricas más destacables provenientes del sur de Francia. ${ }^{2}$ Se trata de un paralelepípedo de piedra arenisca que fue hallado a

1 Trabajo realizado en el marco del Proyecto de Investigación FFI2012-36069-C03-01 del Ministerio de Economía y Competitividad y del Grupo IT698-13 del Sistema Universitario Vasco, por una parte, y en el marco del programa Marie Sklodowska-Curie (European Union's Horizon 2020 research and innovation programme, grant agreement No 655938), por otra parte. Agradecemos a los miembros de la "Association Culturelle Archéologique et Paléontologique (ACAP)" su amable recibimiento en el Musée de Cruzy para realizar la autopsia y estudio de la pieza el día 25 de noviembre de 2015. Asimismo agradecemos las sugerencias realizadas por los informantes del manuscrito en el proceso de evaluación.

${ }^{2}$ Esta inscripción aparece recogida con la referencia HER.02.374 en la base de datos online Hesperia y con el número B.11.1 en el suplemento de los $M L H$ de Untermann (inédito). 
mediados del siglo xx en la ladera septentrional del oppidum de Ensérune, en circunstancias de las que, desgraciadamente, casi nada se conoce. La pieza se conservó durante décadas en el domicilio particular de su descubridor, M. René Marin, vecino de Ouveillan (Aude), hasta que en 1996 fue donada al Musée de Cruzy, donde hoy en día se conserva.

En el otońo de 1997 Jürgen Untermann fue directamente informado de la existencia de la pieza mediante carta de Jean Valdeyron, quien le proporcionaba, asimismo, una fotografía de la que Untermann se serviría para un primer estudio informal de la inscripción: "Ci-dessous je propose une transcription des lettres ibériques qui se base sur votre excellente photographie, mais vous saurez que la plus belle photo ne peut substituer l'autopsie». ${ }^{3}$ Basándose en las primeras observaciones que

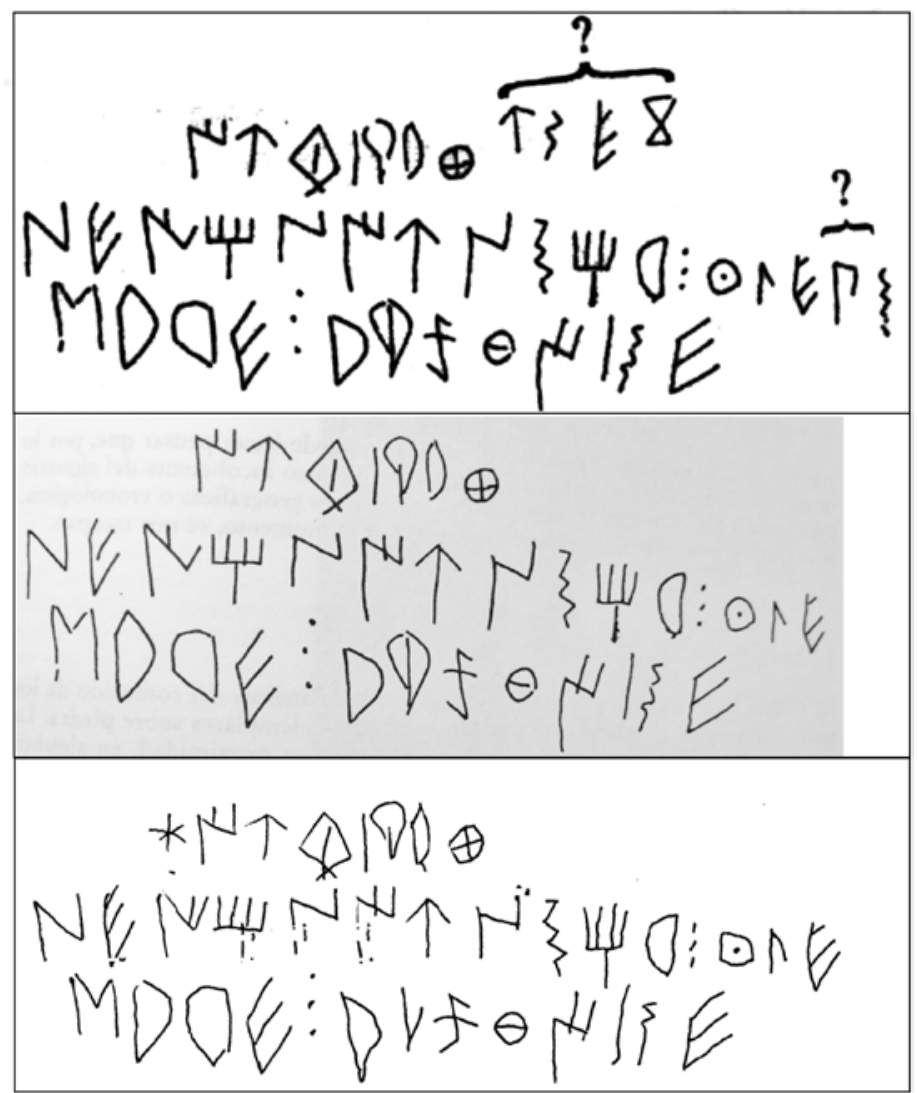

Figura i. Dibujos publicados. Arriba: Valdeyron (1998) y Untermann (1999). En medio: Velaza (2001). Abajo: Untermann (2002)

3 Damos también las gracias a la A.C.A.P. por habernos facilitado la correspondencia epistolar intercam- biada en este período con J. Untermann a propósito de la pieza. 
le proporcionó Untermann, Valdeyron dio a conocer ya en 1998 una primera noticia de la pieza, que todavía no podría ser considerada, sin embargo, como la editio princeps propiamente dicha del texto (Valdeyron 1998). La autopsia del monumento fue llevada a cabo finalmente por Untermann en la primavera de 1998, y su primera edición epigráfica y filológica apareció publicada en Archéologie en Languedoc (23), con numerosas erratas tipográficas que afectaban al ibérico, y en el número 2 de la entonces joven revista Palaeohispanica (Untermann 1999 y 2002 respectivamente).

Este nuevo trabajo dedicado a la piedra de Cruzy nace de una serie de misiones epigráficas realizadas en el sur de Francia por los firmantes de este artículo en el marco del proyecto Hesperia, cuya finalidad es mantener actualizado y revisado, a la luz de los nuevos avances, el corpus de inscripciones paleohispánicas, ejemplarmente publicado por Untermann entre 1975 y 1997 en los Monumenta Linguarum Hispanicarum, en una nueva base de datos online. ${ }^{4}$ La revisión de esta inscripción proporciona, como se verá, una lectura notablemente distinta, que obliga a una reconsideración de la onomástica mencionada en el texto.

\section{DesCripCión DE LA PIEZA}

Como hemos dicho, se trata de bloque de piedra ligeramente rectangular cuyas medidas máximas son: $(31) \mathrm{cm}$ altura $\mathrm{x}(40) \mathrm{cm}$ anchura $\times(29) \mathrm{cm}$ profundidad. El módulo de los signos es reducido, teniendo en cuenta las proporciones de la pieza: en la primera y segunda líneas oscilan entre 2 y $1 \mathrm{~cm}$ y en la tercera oscilan entre 2,5 y $1 \mathrm{~cm}$.

La piedra sólo presenta indicios claros de haber sido cortada por su cara frontal, donde también se ha rebajado toscamente y pulido el campo epigráfico. El aspecto de las otras caras es más de roca que de piedra tallada, hecho por el que la pieza no es identificable con una estela, altar o pedestal, y tampoco claramente con un sillar, columna o cualquier otro elemento arquitectónico definible. Los signos se corresponden también con este aspecto poco monumental, aunque cabe destacar que su incisión es profunda, probablemente realizada con martillo y cincel, y no simplemente esgrafiada con un instrumento de punta seca, como sería el caso de la epigrafía parietal o rupestre, de la que se conoce un importante conjunto en la cercana Cerdaña pirenaica (Campmajo y Untermann 1991; Campmajo y Ferrer i Jané 2010; Ferrer i Jané 2010; 2013; 2014; 2015). Sea como sea, su factura no parece tampoco ser producto de una officina epigráfica especializada, como sí sucede con otras inscripciones monumentales sobre piedra conocidas en el mundo ibérico, especialmente ya en época romana (Velaza 2002a, 56-61).

Esta circunstancia encaja con su cronología, ya que su paleografía es característica de los siglos v-III a.C., tal como se analiza en el apartado siguiente. Otro ejemplo de la escasa epigrafía ibérica sobre piedra anterior a época romana es un bloque inscrito del Puig de Sant Andreu d'Ullastret que se encontró reaprovechado en una construcción (Zona 19-Sector 2-Mur 3) de principios del siglo IV a.C., por lo que necesariamente debe ser de un momento indeterminado anterior (Casas et al. 2002, 246; Untermann 2002a).

\footnotetext{
${ }^{4}$ La base de datos Hesperia es accesible en: http:// hesperia.ucm.es/
} 
En consecuencia, la morfología poco común de la pieza y de la inscripción, así como la falta de noticias precisas sobre el contexto del hallazgo, hacen imposible determinar la funcionalidad del monumento y del texto que contiene. No obstante, Untermann $(2002,355)$ sugería que pudiera tratarse de un sillar de la muralla de Ensérune o de un edificio público (Untermann 2005, 1095); Velaza $(2001,651)$ se inclina por catalogarla entre el grupo de inscripciones rupestres, considerando que se trata de una escritura espontánea sobre un bloque de construcción, mientras que De Hoz $(2011,380)$ considera la posibilidad de que fuera una estela rústica. A pesar de su aspecto tosco y de que Untermann la descarte como estela por su grosor (Untermann 2002, 355), sus dimensiones, $40 \times 29 \mathrm{~cm}$, no son muy distintas en anchura y profundidad de las de las estelas de Badalona C.8.10* (= BDH, B.03.01) de 48,5 $\times 26 \mathrm{~cm}$, C.8.11* (= BDH, B.03.02) de $42 \times 27 \mathrm{~cm}$ y C.8.1 (= BDH, B.04.01) de $41 \times 29 \mathrm{~cm}$ (Comas et. al 2002; Simón 2013, 137). De hecho, estas dimensiones las hacen compatibles también con los sillares de las murallas, donde algunas de ellas han sido reaprovechadas, como es el caso de la estela perdida de Barcelona (C.9.1 = BDH, B.05.01).

Conviene recordar, por último, que las inscripciones ibéricas sobre piedra - con excepción de la ya mencionada epigrafía rupestre — son extremamente raras en la región; de hecho, el tipo más común de inscripciones ibéricas sobre soporte pétreo, esto es las estelas funerarias, no cuenta por el momento con ningún ejemplar que provenga del territorio francés.

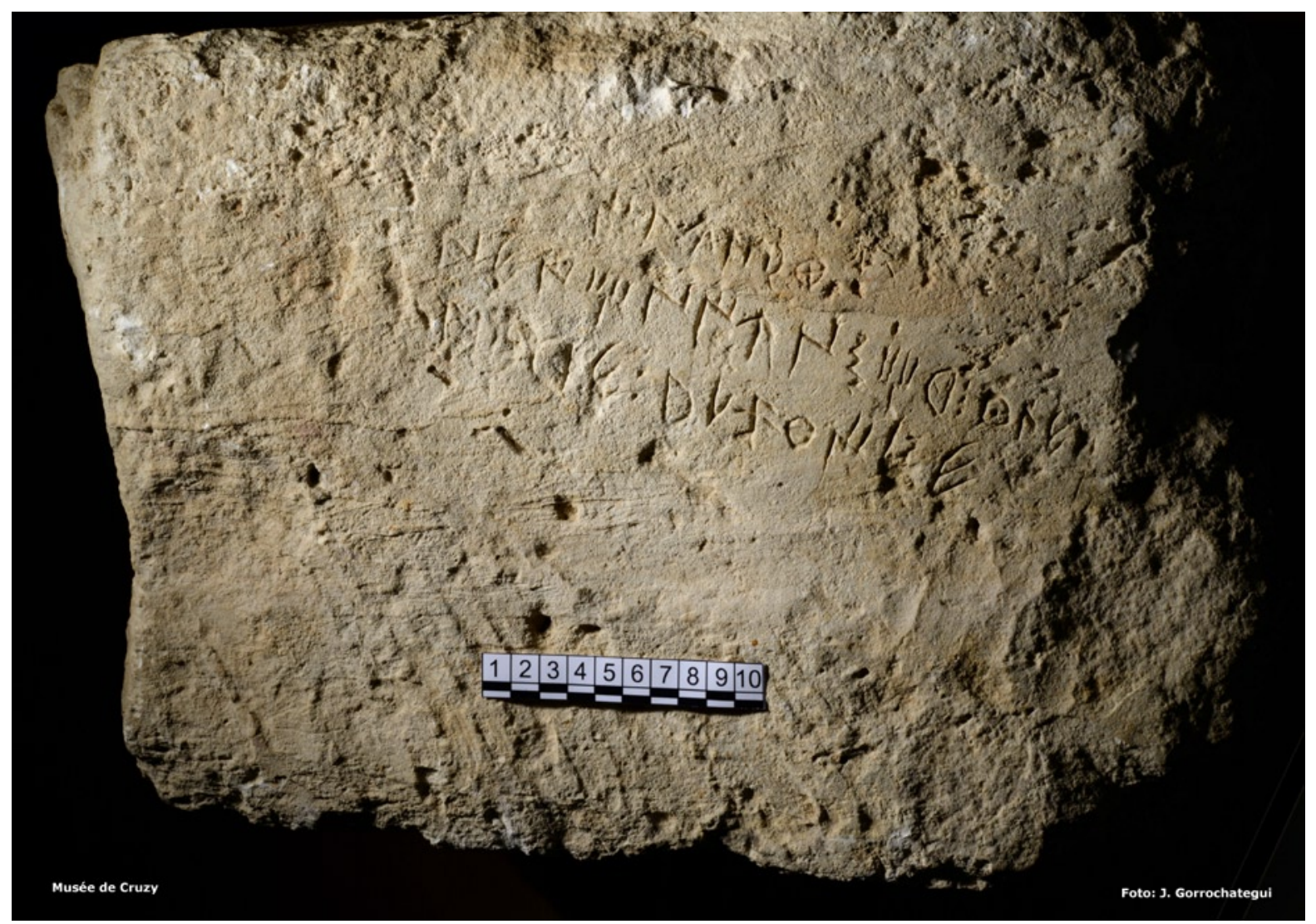

Figura 2A. Fotografía de la piedra de Cruzy 


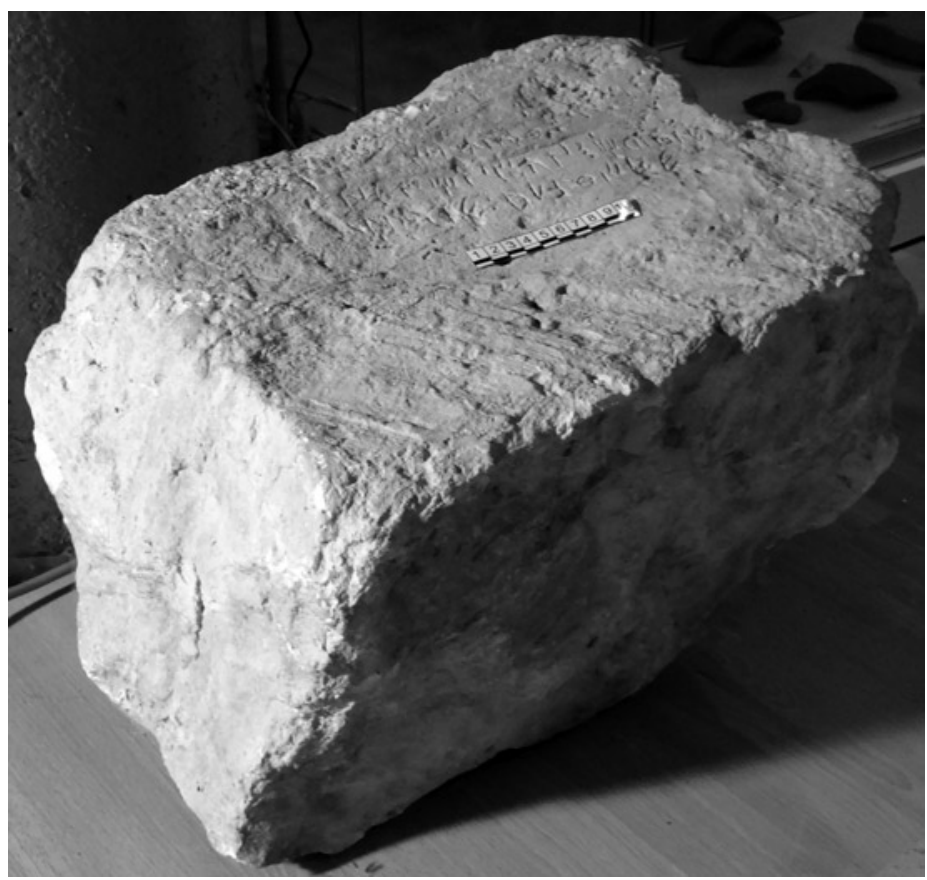

Figura 2B. Fotografía de la piedra de Cruzy

\section{Edición DEL TEXTO Y COMENTARIO PALEOGRÁFICO}

De esta inscripción se han publicado tres dibujos distintos (Fig.1), el primero por Valdeyron (1998) coincide con el publicado por Untermann en 1999, que aunque no se indica explícitamente, aparentemente es obra de Valdeyron, mientras que el publicado por Velaza (2001) — que atribuye a Untermann - coincide con el anterior, excepto en el hecho de que ya no se representan los signos adicionales de las dos primeras líneas, marcados por un interrogante en el primer dibujo. El último dibujo publicado es el Untermann $(2002,356)$, en el que explícitamente (n. 2) indica que es una adaptación del de Valdeyron en el que se han suprimido los signos dudosos, aunque presenta a su vez otras diferencias con los anteriores: la presencia de un posible signo bo inicial en la primera línea, (bo?)iubebaŕete, la representación como signo ḿ del segundo signo del último segmento del texto, que en los dibujos anteriores aparece como un signo ́́, y por último el dibujo del trazo característico del signo $5^{5}$ de la primera línea.

A partir de los dibujos y fotografías publicadas, otros investigadores han propuesto diversas variantes de lectura. Por lo que respecta a los lugares con la existencia de posibles signos, Faria (2005, 168) considera que existe un signo perdido al final de la segunda línea, kule[-]śare, y Simón (2014, 153) considera la primera línea quizás incompleta por la izquierda, [---]iubebaŕete. Respecto del segundo signo del último segmento del texto, dibujado como un signo ḿ por Untermann en 2002, fue transcrito sin embargo como $\mathbf{r}$ (sin diacrítico), quizás por error, puesto que las $\mathbf{r}$ de este texto

5 Citamos según la catalogación de los alógrafos de la tabla proporcionada por Untermann en $M L H$ III.1. 
están orientadas en sentido contrario, mientras que en los dibujos anteriores este signo se dibuja como un signo ŕ. Silgo $(2004,196)$ ha sido el único que ha considerado la alternativa de lectura ḿ, aḿkiteibase, mientras que Faria $(2002$, 127) lo considera no legible; el resto de los estudiosos se ha dividido entre optar por r, arkiteibase (Velaza 2001, 651; Silgo 2004, 196; Orduña 2006, 229) o por ŕr, aŕkiteibase (Correa 2004, 80; Rodríguez Ramos 2005, 120; Moncunill 2007, 85; Simón 2014, 153). Otro signo problemático es el penúltimo signo de la primera línea que a pesar de ser dibujado como un signo a en todos los dibujos, con el apéndice de a 5 en el dibujo de Untermann del 2002, es transcrito por Untermann inexplicablemente como un signo e, circunstancia que otros investigadores ya habían propuesto corregir (Faria 2002, 127, iubeba[-]ate; Correa 2004, 80, iubebaŕate; Rodríguez Ramos 2005, 120, iubebaŕate). A partir de las fotografías publicadas, Faria $(2002,127)$ no considera legibles el penúltimo y el antepenúltimo signos de la última línea, a[-]cidei[-]e, ni tampoco los dos supuestos signos ŕ de iubeba[-]ate y a[-]cidei[-] e.

Con los matices ya indicados, la lectura más habitual de esta inscripción era la siguiente:

\section{iubebaŕete (o iubebaŕate) \\ neitiniunstir : kule \\ śare : aŕlkiteibase}

Por lo que respecta a la autopsia realizada, las dudas de lectura afectan al cuarto signo de la primera línea; aunque está parcialmente erosionado en su parte superior, no parece que pueda ser otra cosa que un signo $\mathbf{n} 1$. También dudoso es el segundo signo de la tercera línea, puesto que casi ningún trazo puede darse por seguro, aunque los restos perceptibles son compatibles con un signo a5. El trazo inferior característico del signo a5 es claro en el quinto signo de la primera línea y algo más tenue en el primer signo del segundo segmento de la tercera línea. Los signos antepenúltimo y penúltimo de la tercera línea están erosionados en su mitad inferior, pero parece claro por la parte conservada que difícilmente pueden ser otra cosa que ba1 y s9/10.

Aunque algunas variantes del signo tu pueden presentar formas similares a las del signo $\mathbf{u}$, al carecer de base, como ocurre con el signo tu de una de las fusayolas de Oliete, no parece que pueda ser el caso del signo u de la primera línea este texto, — que de leerse tu permitiría identificar uno de los formantes antroponímicos más frecuentes: iltun $(M L H$ III.1 $\$ 7.62)$ 一, puesto que no se aprecian diferencias significativas entre este signo y el signo u del elemento iunstir.

Respecto de las dudas expresadas en los dibujos publicados, no se confirman los cuatro signos dudosos del final de la primera línea ni los dos dudosos del final de la segunda línea del dibujo de Valdeyron (1998). Tampoco se confirma el signo bo 2 inicial dudoso que dibuja y transcribe entre paréntesis Untermann $(2002,355)$. El signo ḿ4 de la tercera línea es claro, de profunda incisión y no afectado por erosión ninguna, por lo que no tiene sentido la lectura como ŕ 7 , ni pensar en un incompleto $\mathbf{r} 5$, dado que en este texto se usa sistemáticamente $\mathbf{r} 3$.

El texto se distribuye en tres líneas sobre un campo que ha sido preparado someramente mediante el alisado de la piedra para recibir la inscripción. Se percibe una separación entre la primera línea y las dos inferiores, que quizá indique que el campo fue agrandado en la parte superior a partir de un alisamiento previo. En la parte derecha, a la altura de las líneas 2 y 3, se perciben restos de la línea de demarcación del campo. Las líneas 2 y 3 están justificadas al margen izquierdo, llegando la línea 2 hasta el extremo derecho del campo fijado, mientras que el texto de la inscripción termina en la línea 3 antes de llegar al borde del campo. La línea 1, que presenta signos regularmente más pequeños que los de las líneas inferiores, está justificada al centro.

El signario empleado es el ibérico nororiental en su variante dual, tal como se desprende de la presencia de un signo te complejo en la l. 1 (te14) y de otro signo te simple (te8) en la l. 3, así 

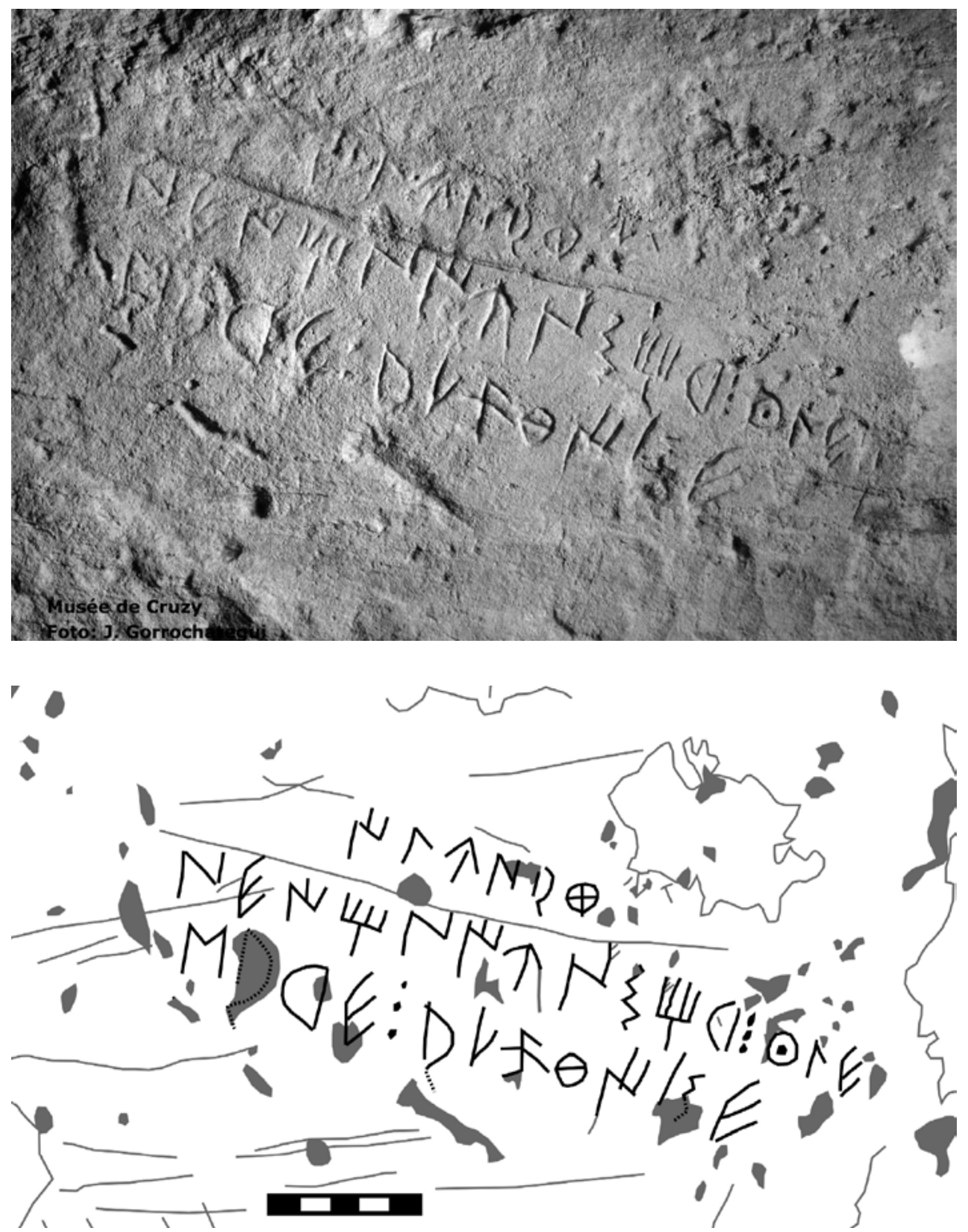

Figura 3. Dibujo de la inscripción y fotografía del campo epigráfico

como del uso de los signos ti y ki complejos (ti7) y (ki3). El uso del signo a5 es típico de las inscripciones de la región y los signos e4, ku2 y s9/10 son característicos de las inscripciones más antiguas. Las interpunciones empleadas consisten en tres puntos verticales. A pesar de que las inscripciones ibéricas nororientales susceptibles de haber usado el sistema dual son unas 700, de un conjunto de más de 2.000, sólo unas 30 presentan dualidades explícitas (Ferrer i Jané 2015a), siendo esta inscripción una de ellas al presentar la dualidad del signo te. El uso del signario dual es un indicio favorable a considerar que la cronología de la pieza corresponde a los siglos v-III a.C., periodo en el que se sitúan la práctica totalidad de las inscripciones duales (vid. Ferrer i Jané 2005, 967-973). 
Así pues, la nueva lectura que proponemos para el texto es la siguiente:

\author{
ilunate \\ neitiniunstir : kule \\ śare : ańkideibase
}

\title{
4. ANÁLISIS LÉXICO
}

ilunate: esta forma, anteriormente leída mayoritariamente como iubebaŕete, había sido considerada como la adaptación al ibérico de un nombre personal galo (Untermann 2002, 357; Rodríguez Ramos 2014, nota 6) o como un nombre ibérico (Moncunill 2007, 195), propuestas que con la nueva edición del texto quedan invalidadas. La interpretación más probable parte de la identificación de un antropónimo iluna, segmentable en ilun y a, seguido del morfo te.

La raíz ilun está presente en ibérico en casos como iluntorka (F.20.2,B-3) y uśtalarilune (F.9.5,5); probablemente también en ilunbaŕbi (G.1.4), tal como sugiere Orduña $(2011,15)$ en el segmento leído iltunbaŕa por Untermann en MLH. En cambio, su presencia en kebelsilunin (F.9.8,A-6) y en ]silunini[ (C.0.3,2) depende de la segmentación correcta, puesto que alternativamente podría identificarse el formante antroponímico unin $(M L H$ III.1 \$7.139). Es dudoso que pueda identificarse en el texto leído habitualmente ]ilun[ en escritura meridional en un fragmento de cerámica pintada de Meca, puesto que sería mejor la lectura ]ikaun[ (Ferrer i Jané et al. 2015); asimismo, la alternativa de lectura á́beiluna[ para el plomo G.6.1 no parece la más probable en un texto de lectura muy dudosa.

A pesar de las dudas en la segmentación y en la interpretación como antropónimos de la mayor parte de los ejemplos anteriores — solo para iluntor parece razonablemente plausible por el contexto-, el elemento ilun es considerado de forma general una variante del formante antroponímico iltun (MLH III.1 \$7.62) (de Hoz 1997, 142; Faria 2003, 227; Orduña 2006, 344; Moncunill 2007, 351; Rodríguez Ramos 2014, 24). Puede citarse aquí la debatida cuestión de la grafía del formante en el Bronce de Ascoli bajo la forma ILLVN, que se ha visto como recurso gráfico, al igual que el uso de la secuencia lt, para la expresión de una segunda lateral que no tuvo signo propio en el signario ibérico. No es imposible, por consiguiente, que este hecho sea la causa de una cierta vacilación gráfica entre ilun e iltun.

La identificación de ilun y del morfo te aislaría un elemento final a que no obstante no es un elemento característico de la antroponimia ibérica, ni como segundo formante ni como elemento de enlace entre la raíz y el sufijo. La existencia de un morfo a podría deducirse de su uso asociado al antropónimo balkeśiŕ en el plomo en escritura ibérica suroriental de La Carencia (Velaza 2013) con la presumible función de marcar al destinatario o propietario de la carta, ya que aparece como texto único en el exterior visible de la lámina de plomo plegada. No obstante, podría tratarse de una forma local del morfo -ar presente también en la misma posición en el plomo de Alcoi (G.1.1): sakarisker-ar-nai. En cambio, con más claridad puede presumirse la existencia de un morfo a en el elemento baneia (G.7.5, A), en el que Ferrer i Jané (2006, anexo 3) identifica un morfo ei característico de posibles antropónimos formados con base numeral, ban en este caso, como quizás biei (F.20.1) y sorsei (C.1.8), y que permitiría aislar un morfo a que en el texto del plomo G.7.5 estaría coordinando dos segmentos consecutivos: teia • baneia (Moncunill 2007, 108 y 302). El morfo -a también ha sido propuesto como formante característico de topónimos por Faria $(2008,87)$, aunque ninguno de los casos indicados presenta una segmentación especialmente clara: bersa, EGARA, śigaŕa, etc. 
El único paralelo para iluna como tal nombre personal es controvertido: [Ill] una en una inscripción latina perdida de Iruña de Oca (CIL II, 2936 = CIL II, 5815). De estar bien reconstruido puede entenderse sin problemas como acomodación a un nombre de tema en - a latino, por tratarse de nombre de mujer. El frecuente nombre de divinidad, atestiguado como Ilunno, Ilunni en los altares aquitanos (Gorrochategui 1984, 333), remite a Ilunn, sin rastro de vocal final $-a$.

El morfo -te es un sufijo muy productivo en ibérico al que probablemente hay que atribuir el valor de agente, tal como se acepta de forma mayoritaria (Correa 1994, 282; Velaza 2002; Rodríguez Ramos 2005, 44; Untermann 2005, 1143 ; Quintanilla 2005, 517; Ballester 2005, 387; Ferrer i Jané 2006, Anexo 12; Moncunill 2007, 300), por su aparición en la fórmula NP-te+ekiar, ${ }^{6}$ a la que se da un valor semántico parecido al latín NP+fecit, aunque otros investigadores manifiestan ciertas dudas (Orduña 2006, 305, 2009, 510; de Hoz 2011, 269).

Este sufijo complementa también nombres personales que aparecen asociados a la expresión iunstir, o alguna de sus variantes, como sería nuestro caso. Uno de los paralelos más cercanos se encuentra en el texto del ponderal de Calafell (C.41.1Sup = BDH, T.05.01), que ofrece una estructura comparable a la del texto de Cruzy (vid. también infra el análisis de neitiniunstir) y donde un sufijo -te parece también claramente aislable tras nombre personal y abriendo el texto: agirukeŕ-te iustir baśbeŕ agirórdin uldiladie. ${ }^{7}$

Una interpretación menos probable para ilunate consiste en analizarlo como un compuesto formado por ilun más otro formante ate, que tampoco contaría con paralelos claros en el repertorio antroponímico ibérico, ya que los dos casos aducibles, kaŕate (B.1.33,2 = BDH, HER.02.033) o śuate (B.1.67 = BDH, HER.02.067), admiten explicación plausible como adaptación de nombres galos (cf. BDHesp).

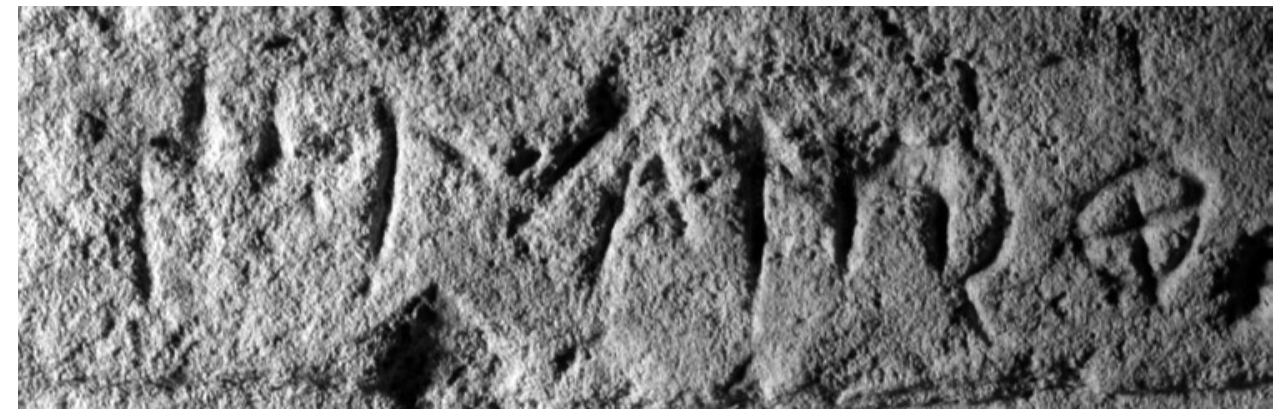

Figura 4. Detalle de la l. 1: ilunate

\footnotetext{
6 Sin embargo, en los textos duales el sufijo suele aparecer con la variante sonora (NP-de egiar), a diferencia de lo que ocurre en nuestro caso, así como en otros testimonios de NP-te iunstir (vid. nota siguiente). A pesar de ello, suele considerarse que -te y -de podrían ser variantes de un mismo sufijo. La alternancia podría deberse a imprecisiones en el uso del dual o incluso al contexto fonético (téngase en cuenta, por poner un ejemplo de la complejidad de la cuestión, que el silabario ibérico no permite escribir la oclusiva en posición fi-
}

nal, de modo que este sufijo podría en realidad ser -t o -d y ser objeto de ensordecimientos o sonorizaciones en función de la naturaleza vocálica o consonántica del sonido siguiente). Finalmente, recuérdese también la probable variante $\mathrm{NP}+\boldsymbol{t a}+(\boldsymbol{e}) \boldsymbol{g i a r}$ (Ferrer i Jané 2005, 963; 2008; Moncunill 2007, 298) con sorda.

7 Otros casos de NP-te iunstir son: betukine-te iuśdir (F.17.2,B.a), sakaŕakin-de iuśdir (F.17.2,A-1), saltutibai-te iumstiŕ (F.13.5), y quizás también Jikamteiunsr (Ferrer i Jané - Escrivà 2013). 
neitiniunstir: fórmula bien conocida en ibérico (vid. para un análisis de conjunto Moncunill 2007, 249), que se documenta tanto en scriptio continua como con una interpunción separando los dos elementos: neitin y iunstir. La heterogeneidad de soportes en los que se documenta ${ }^{8}$ dificulta, sin embargo, su interpretación. De hecho, tanto un elemento neitin como un elemento iunstir son susceptibles de actuar como formantes antroponímicos (cf. Neitinbeles [CIL II 6144], en una inscripción latina de Terrassa y iunstir-laku [F.9.5]). Por consiguiente, neitiniunstir podría corresponderse también, al menos en teoría, con un antropónimo. Existen, con todo, distintos argumentos para descartar esta opción: en primer lugar, debe tenerse en cuenta su relativamente frecuente aparición, que sería anómala dentro de la tendencia general de uso de los nombres ibéricos, en el que el índice de repetición es más bien bajo; en segundo lugar, sería difícil explicar por qué los formantes del supuesto antropónimo aparecen a veces separados por interpunción; en tercer lugar, el ejemplo de la piedra de Cruzy, en el que neitiniunstir podría ir detrás a un nombre seguido de un sufijo -te, haría preferible una interpretación alternativa a la de nombre personal, y abriría las puertas a un análisis como verbo o sustantivo verbal. ${ }^{9}$ Por ello, es preferible una interpretación de neitiniunstir como fórmula de contenido verbal para la que, atendiendo a sus variados contextos de aparición, una interpretación en el sentido de fórmula de salutación (De Hoz 1979, 236; Velaza 1991, 80-81, Orduña 2009, 507) o propiciatoria (Rodríguez Ramos 2005, 58) nos parece efectivamente plausible.
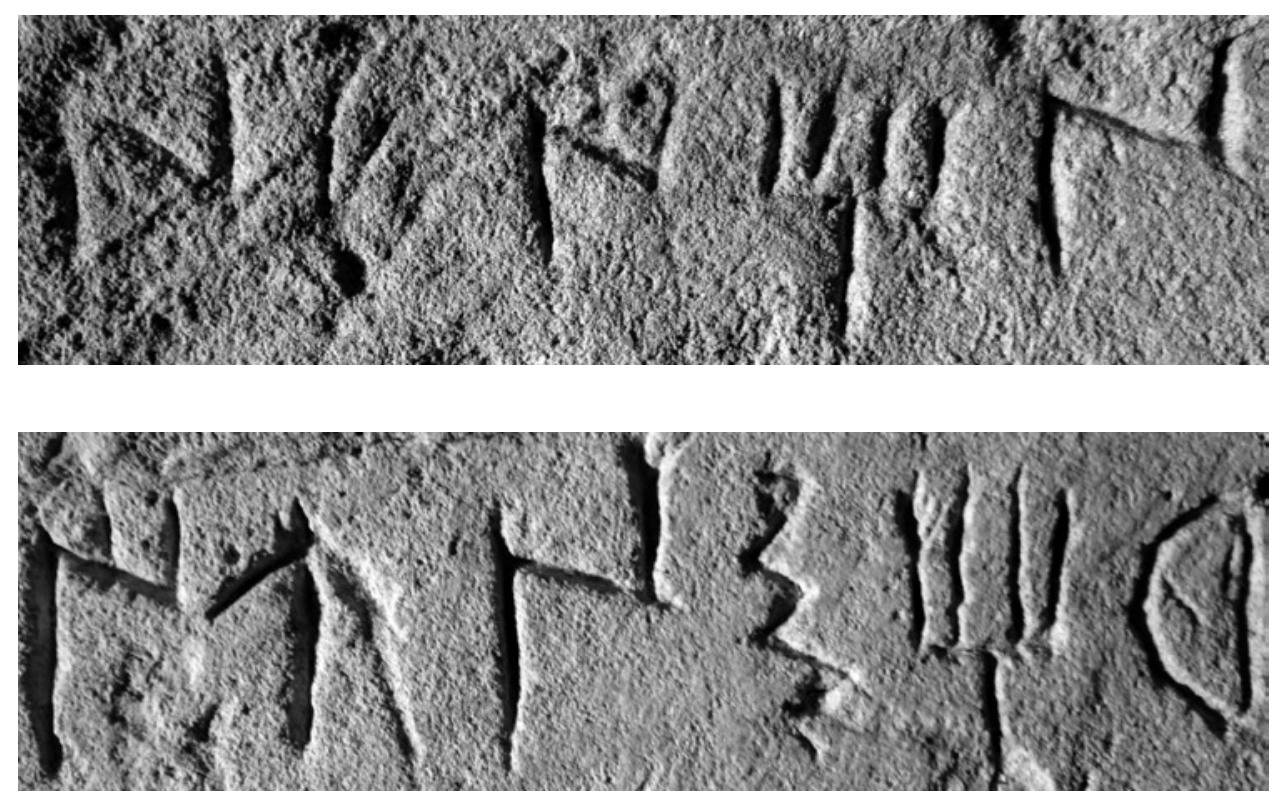

Figura 5. Detalle de la l. 2: neitiniunstir

8 Por el momento ha aparecido en: 1. tres plomos (el plomo de la colección Marsal, en el que aparece encabezando dos de sus cuatro textos, una vez con interpunción interna, y la otra en scriptio continua; en la última línea de uno de los plomos de Tivissa [C.21.10Sup = BDH, T.09.10]; y muy probablemente también en el inicio de uno de los plomos de Empúries [C.1.24.Sup. (= BDH, GI.14.23)]); 2. el askos de Ullastret (C.2.8,1 =
BDH, GI.02.08), inscripción de lectura muy dudosa, pero donde esta fórmula parece también encabezar el texto; 3. finalmente, podría documentarse también en la estela de La Vispesa Binéfar, en la forma incompleta neitin[ (D.12.1,A). También se documenta en una inscripción rupestre inédita de Ger (BDH, GI.02.13) aún en curso de estudio (Ferrer i Jané e.p.), aunque en este caso aparecen separados por un tercer elemento. 
Por otra parte, el elemento iunstir con sus variantes gráficas o fonéticas (uśtiŕ, unsir, iunsir, iumstir, iustir, iuśdir y iunśdir), que se documenta de forma aislada repetidas veces en ibérico, es posible que actúe en algunos casos como la versión reducida de la fórmula neitiniunstir (vid., por ejemplo, Ferrer i Jané 2006, anexo 16). Uno de los ejemplos más elocuentes en este sentido es el ya mencionado texto del ponderal de Calafell (C.41.1Sup = BDH, T.05.01), con una estructura muy parecida a la del texto de Cruzy, en la que el primer nombre podría corresponderse con el dedicante, a continuación seguiría la descrita expresión formular, y a continuación seguirían el destinatario o destinatarios de dicha dedicatoria.

kuleśáre: Tal como propuso ya Untermann (2002), parece conveniente unir el final de la 1.2 con el inicio de la línea siguiente, con lo que se obtiene un nombre personal formado con los elementos kuleś (MLH III.1, \$7.80) y śar (cf. ildiŕśar [B.7.34,19= BDH, AUD.05.34] (Solier 1979), ikośar [D.0.1,B-2 = BDH, SP.01.06] (Untermann 1989) y quizás katuiśar [B.1.20 = BDH, HER.02.020]), y sufijado con el morfo -e (Untermann 1990 = MLH III.1, 163; Orduña 2006, 61; Moncunill 2007, 149-150; Ferrer i Jané 2006, anexo 2). Si asumimos que el sufijo -te asociado al primer nombre encaja bien con la marca del agente de la acción, podríamos postular que -e se corresponda con la marca del destinatario de dicha acción (Silgo 1994, 151; Faria 2006, 117; Velaza 2013, 542). Esta interpretación del valor del sufijo encajaría bien con algunas inscripciones que presentan como texto único un antropónimo seguido de tal morfo (como por ejemplo bintaŕ-e (C.1.7 = BDH, GI.14.07), en una plaquita en forma de etiqueta, o egeŕśor-e (B.23.13,A = BDH, PYO.07.13), en una de las inscripciones rupestres de la Cerdanya (Campmajo y Ferrer i Jané 2010), para la que una interpretación como dedicatoria también parece adecuada. Otros antropónimos sufijados con e y que podrían ser destinatarios de la acción de (neitin)iunstir son uldiladi-e del ponderal de Calafell (C.41.1Sup = BDH, T.05.01) y balkeśír-e del plomo de La Carencia, ya citados.
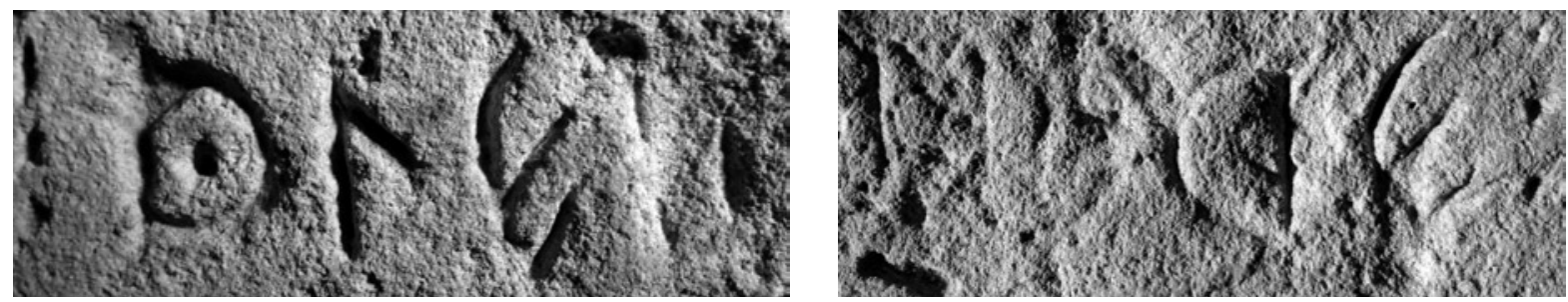

FIgURA 6. Detalle l. 3/4: kuleśare

aḿkideibase: la segmentación de esta forma podría ser aḿ-kidei-bas-e o incluso aḿ$\boldsymbol{k i d e - ( e ) i - b a s - e , ~ p e r o ~ s u ~ a n a ́ l i s i s ~ n o ~ r e s u l t a ~ e n ~ a b s o l u t o ~ c l a r o . ~ L a ~ o p c i o ́ n ~ m a ́ s ~ s o c o r r i d a ~ s e r i ́ a ~ i n t e r - ~}$ pretarlo como un nombre personal trimembre (án-kidei-bas) sufijado con -e; o como un nombre bimembre (án-kidei) seguido de un elemento pronominal -bas y sufijado también con -e. La tercera posibilidad, sin duda más sugerente y cargada de consecuencias, consistiría en interpretar $\boldsymbol{a} \boldsymbol{a} \boldsymbol{k i d e i}$ como una forma apelativa seguida del mencionado pronombre bas y del sufijo - $\boldsymbol{e}$.

\footnotetext{
9 Es posible que el núcleo verbal de neitinsiunstir se encuentre propiamente en iunstir, como ya fue sugerido por Untermann $(1987,45-7)$, aunque le diera el sentido de oportet o licet; neitin, por su parte, podría
}

ser algún tipo de complemento estrechamente relacionado con esta palabra de contenido verbal, una especie de objeto con el que parece configurar una expresión compleja. 
El análisis como antropónimo partiría de la identificación de un primer formante aú que podría ser una variante del formante antroponímico an (MLH III.1 \$7.10), que al menos en dos ocasiones aparece anotado como anḿ: anḿbeŕai [F.9.7,B-5] y anḿkitare [D.18.1,C-2 = BDH, B.26.01] ${ }^{10}$.

En la segunda posición, sería entonces esperable otro formante antroponímico kidei o kide seguido de un morfo ei (Ferrer i Jané 2006, anexo 3), pero la interpretación de esta secuencia como antropónimo es menos clara. Para kide podríamos traer a colación ban-kite (D.18.1,B-5; D.18.1,C-4 = BDH, B.26.01; E.4.2) o kelse-kite (E.4.2), aunque la interpretación de estas dos formas como nombres de persona es discutible (Moncunill 2010, 56). De hecho, los diversos elementos con base kita/kite (kite, kitar, kiterter, kitei) parecen formar parte del léxico metrológico ibérico, dada su asociación a posibles numerales y su presencia en las marcas de valor de las monedas (Orduña 2005, 491; Ferrer i Jané 2009, 463). En concreto el elemento kidei es claramente aislable en otros texto ibéricos donde su aparición junto a posibles numerales (kiteibors, F.20.1.A), y sobre todo a la palabra śalir (G.1.1: binikebin - śalir - kidei), invita a un análisis de la forma como "elemento cuantificado", solución que en principio no parece adecuada en nuestro caso ${ }^{11}$. El morfo -ei, además de su aparición tras elementos del contexto metrológico, también aparece asociado, o quizás ya integrado, como parte del antropónimo en otros claros nombres de persona como alotikeŕ-ei (C.3.1) o balkebeŕ-ei (F.13.6). La presencia, pues, de este morfo podría ser un argumento a favor para considerar kide-(e)i como un elemento antroponímico, aunque, como decíamos, su raíz no cuenta con paralelos que apunten en este sentido.

El elemento bas admitiría, ahora sí, una interpretación como formante de nombres personales ( $M L H$ III.1, \$7.27, como en aiubas A.33). Sin embargo, la existencia de antropónimos trimembres en ibérico es dudosa, hecho por el que no descartaríamos una interpretación de este elemento como forma pronominal, función que ha sido sugerida por algunos investigadores (Orduña 2006, 170; Ferrer i Jané 2006, 153, n. 91; Artigues et al. 2007, 244).

Sea como sea, el análisis de ánkideibas o aḿkidei como nombre de persona no resulta plenamente convincente, tanto por su estructura trimembre, como por la falta de paralelos antroponímicos para el elemento kidei. Lo que sí parece seguro es que en el final de la secuencia el sufijo -e debería ser el morfo ya analizado y que haría concertar el conjunto de la forma con el antropónimo anterior kuleśár-e.

Alternativamente, aḿkidei podría ser analizado como un término del léxico común —quizás internamente también segmentable en varios elementos, án-kide-(e)i-, que expresara la relación existente entre los dos antropónimos citados en primer lugar, iluna y kuleśar, y cuyo sentido no nos sería por el momento posible determinar con exactitud. A este posible apelativo le seguiría la mencionada forma pronominal bas (como podría ser en latín huius, eius o incluso suus), con la función de conectar el apelativo con el nombre al que éste hace referencia.

Esta interpretación vendría principalmente apoyada por argumentos tipológicos, puesto que en otras tradiciones epigráficas antiguas, como la griega o la latina, son abundantes las dedicatorias con una estructura semejante (esto es: "esta persona a esta persona, su X").

10 Teniendo en cuenta que el grafema ḿ tiene probablemente el valor fonético de /na/ (así parece desprenderse de la adaptación del sufijo -mín como -nai en alfabeto grecoibérico), un supuesto formante am podría también ser puesto en relación con ana, documentado en anabedi en uno de los plomos de La Carencia (Velaza 2013, 542) y quizás también en ]anako (D.4.2 = BDH, B.33.02).
11 No obstante, en algunos textos se documentan posibles numerales que parecen funcionar con iunstir, como sería el caso de baŕbin en F.9.7.A, F.9.7.B y F.17.2.A (Ferrer i Jané 2009, 479) y de la rupestre inédita de Ger [BDH, GI.02.13] (Ferrer i Jané e.p) donde aparece el elemento kite en la secuencia iunstir·baŕbinkite. 
Dentro de la propia epigrafía ibérica una construcción parecida ha sido también identificada en el texto del colgante de Can Gambús (Artigues et al. 2007, $244=$ BDH, B.42.01): deśailaur beŕsír[teba]n bitan astebeibaseban. Según una de las interpretaciones planteadas por los editores, los distintos antropónimos presentes en el texto podrían corresponderse, a pesar de la ausencia de sufijos, con el dedicante y el beneficiario, cuya relación sería explicitada por un apelativo, en este caso el conocido eban, que para algunos investigadores tendría el sentido de 'hijo' (Velaza 1994 y $\left.2004^{12}\right)$. En relación con este supuesto apelativo encontraríamos también en este caso el elemento bas, aunque aquí precediendo a eban, a diferencia de lo que ocurre en nuestro caso, donde bas sigue a ánkidei. ${ }^{13}$ Atendiendo, pues, estrictamente a la posición del elemento bas, sería astebei el que ocuparía la posición equivalente de ánkidei, circunstancia que ańadida a la presencia del posible morfo ei en ambos elementos, permitiría pensar en una alternativa interpretativa para el texto de Can Gambús, en la que astebei tuviese la misma función que ánkidei en la de Cruzy.

\begin{tabular}{ccccccc}
\hline NP & Patronímico & Apelativo & Verbo & NP & Pronombre & Apelativo \\
\hline deśailauŕ & beŕsir & {$[$ teba $] n$} & bitan & astebei & bas & eban \\
\hline
\end{tabular}

Esquema interpretativo de la inscripción del colgante de Can Gambús, según una de las propuestas de los editores (Artigues et al. 2007).

Otro paralelo posible para la interpretación de ánkidei como apelativo es la forma śalkidei, documentada tres veces distintas en dos de los plomos de Pech Maho (BDH, AUD.5.34, donde aparece sufijada con - $\boldsymbol{k} \boldsymbol{e}$, y BDH, AUD.5.36a, donde va seguida en una ocasión de -te y en otra de $-\boldsymbol{k e}$ ). Por el hecho de que aparece cerca de formas antroponímicas, a veces incluso entre dos antropónimos (BDH, AUD.5.34), ha sido considerada por algunos autores (Orduña 2006, p. 221) como un nombre en aposición o una forma adjetiva en relación con estos nombres personales.
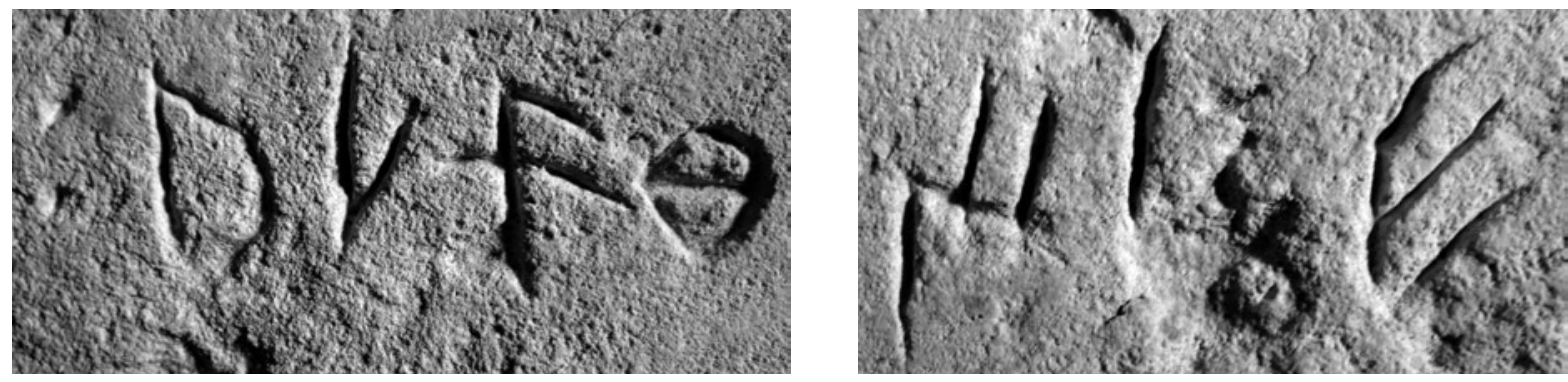

Figura 7. Detalle de la l. 3: ańkideibase

12 En cambio, para una parte de los investigadores esta palabra sería una forma verbal con un sentido similar a curauit (Untermann 1999 = MLH III, 194; Rodríguez Ramos 2001).

13 A juzgar por la presencia de bas en secuencias complejas como basbidiŕbartin (G.1.1), basbiteŕoketine (F.9.7) o basbiteŕo[ (H.0.1) la posición más habitual de este elemento parece ser la proclítica. Sobre la po- sibilidad de que bas pudiera ocupar ambas posiciones, téngase en cuenta que un comportamiento parecido es observable también en otra palabra ibérica a la que se le supone igualmente un carácter pronominal: $-\boldsymbol{m} \boldsymbol{m} \boldsymbol{i}$. Este elemento suele encontrarse en posición enclítica (p. ej. taŕbanikoŕ-ḿi [F.2.2]), aunque existen también algunos ejemplos en posición proclítica (p. ej. mí-ata B.7.14 = BDH, AUD.05.14). 


\section{Conclusión}

En consecuencia, el análisis lingüístico no permite tampoco esclarecer cuál pudo haber sido la funcionalidad exacta de la inscripción, a pesar de que una interpretación como una dedicatoria parece posible.

Así las cosas, la estructura general del texto de la piedra de Cruzy podría encajar en el siguiente esquema:

\begin{tabular}{cccccccc}
\hline NP & SUfIJO & Verbo & NP & SUfIJO & APelativo & Pronombre & SUfIJO \\
\hline iluna & $-t e$ & neitiniunstir & kuleśar & $-e$ & ámkidei & bas & $-e$ \\
\hline
\end{tabular}

El texto se articula alrededor de la secuencia neitiniunstir, seguramente una fórmula propiciatoria, a juzgar por su aparición en textos sobre plomo, algunos de ellos interpretables como cartas. Su sentido, por consiguiente, podría ser similar al latín cura ut valeas, o vale, en su forma reducida (recuérdese la alternancia neitiniunstir / iunstir). La presencia de la secuencia neitiniunstir también en soportes como el askos de Ullastret (C.2.8 = BDH, GI.11.08) o la rupestre inédita de Ger [BDH, GI.02.13] (Ferrer i Jané e.p.) harían esperable que el sentido concreto de esta fórmula fuera, en todo caso, compatible también con el léxico de las inscripciones votivas.

Los nombres que entran en relación a través de esta expresión son, encabezando el texto, Iluna, personaje que podría corresponderse con el dedicante, o el agente de la acción. En la segunda parte del texto aparece mencionado con seguridad otro individuo, Kuleśar, probablemente el destinatario de la dedicatoria o el beneficiario de la acción.

La parte más controvertida en cuanto a su análisis es la última forma, en la que no sería absolutamente descartable que apareciera otro antropónimo, Aḿkidei o Aḿkideibas, a pesar de que no disponemos de apoyos definitivos para tal interpretación. Untermann, de hecho, había ya sostenido que en la parte final aparecieran dos nombres de persona, que él interpretó como nombre + patronímico (Untermann 1999, 357, aunque la lectura para el segundo era errónea: arkiteibas) ${ }^{14}$.

Como solución alternativa, vemos factible que aḿkidei-bas-e pueda ser analizado como una expresión apelativa aḿkidei que describiera la relación existente entre Iluna y Kuleśar, al que seguiría una forma pronominal bas (¿parecida al latín huius, eius, suus?).

En cualquier caso, estas propuestas han de ser tomadas únicamente como unas pautas interpretativas del texto, siendo lo único cierto que su sentido literal nos sigue resultando por desgracia impenetrable.

${ }_{14}$ Sin embargo, la expresión de la filiación en ibérico es un tema sobre el que existe todavía un debate abierto: Untermann $(1995,248 s s$.$) sostuvo que, como$ en este caso, el patronímico pudiera expresarse por la simple yuxtaposición, mientras que Velaza propone que se exprese mediante la palabra eban, o alguna de sus variantes, además de defender que la introducción del nombre del padre en la fórmula onomástica se produjo únicamente bajo la influencia de la epigrafía latina (Velaza 2004), cronología que no encajaría con la de nuestra pieza (siglo III a.C.). 


\section{BiBLIOGRAFÍA}

Artigues, P. Ll., D. Codina, N. Moncunill, J. Velaza, 2007, «Un colgante ibérico hallado en Can Gambús (Sabadell)», Palaeohispanica 7, 239-250.

$\mathrm{BDH}=$ Banco de Datos Hesperia, http://hesperia.ucm.es/

Ballester, X., 2005, «Lengua ibérica: hacia un debate tipológico», Palaeohispanica 5, 361-392.

Campmajo, P., Ferrer i Jané, J., 2010, «Le nouveau corpus d'inscriptions ibériques rupestres de la Cerdagne: premiers résultats» en: Serta Palaeohispanica in honorem Javier de Hoz, Palaeohispanica 10, 249 274.

Campmajo, P., Untermann, J., 1991, "Corpus des gravures ibériques de Cerdagne», Ceretania 1, Arxiu Historic comarcal, Puigcerdà, 39-59.

Casas, S., Codina, F., Margall, J., Prado de, G., 2002, "Noves aportacions al coneixement de l'ampliació nord de l'oppidum del Puig de Sant Andreu (Ullastret, Baix Empordà). Estudi d'una inscripció sobre pedra trobada en aquesta zona», Cypsela 14, 237-250.

Comas, M., Padrós, P., Velaza, J., 2001, «Dos nuevas estelas ibéricas de Badalona», Palaeohispanica 1, 291-299.

Correa, J.A., 1994, «La lengua ibérica», Revista Española de Lingüistica 24:2, 263-287.

—, 2004, «Los semisilabarios ibéricos: algunas cuestiones», ELEA 5, 75-98.

DE Hoz, J., 1979, «Escritura e influencia clásica en los pueblos prerromanos», Archivo Español de Arqueologia $52,227-250$.

—, 1997, «Ficha 226.1», en Rouillard, P. (ed.), Antiquités de l'Espagne, París.

—, 2011, Historia lingüistica de la Peninsula Ibérica en la antigüedad. II. El mundo ibérico preromano y la indoeuropeización. Madrid: CSIC.

Faria, A. M. de, 2002, "Crónica de onomástica paleo-hispânica (3)», Revista Portuguesa de Arqueologia 5.1, 121-146.

—, 2003, «Crónica de onomástica paleo-hispânica (5)», Revista Portuguesa de Arqueologia 6.1, 211-234.

—, 2005, "Crónica de onomástica paleo-hispânica (9)», Revista Portuguesa de Arqueologia 8.1, 163-175.

—, 2008, "Crónica de onomástica paleo-hispânica (14)», Revista Portuguesa de Arqueologia 11.1, 57-102.

Ferrer i Jané, J., 2005, «Novetats sobre el sistema dual de diferenciació gràfica de les oclusives sordes i sonores», Palaeohispanica 5, 957-982.

—, 2006, «Nova lectura de la inscripció ibèrica de la Joncosa (Jorba, Barcelona)», Veleia 23, 129-170.

—, 2008, «Ibèric tagiar. Terrissaires que signen les seves produccions: biứko, ibeitigeŕ, biứbedi i companyia.», Sylloge epigraphica Barcinonensis 6, 81-93.

—, 2009, "El sistema de numerales ibérico: avances en su conocimiento», Palaeohispanica 9, 451-479.

—, 2010, «La llengua i l'escriptura ibèrica a la Cerdanya», Ker 4, 50-59.

—, 2013, "Deux alphabets ibères duals rupestres de Cerdagne», Sources - Les cahiers de l'Âne Ronge 1, 9-18.

—, 2014, «Deux nouveaux alphabets ibères rupestres de Cerdagne», Sources - Les cahiers de l'Âne Rouge 2, 11-20.

—, 2015, "Le nouveau corpus d'inscriptions ibériques rupestres de la Cerdagne : deuxième parution», Sources - Les cahiers de l'Âne Rouge 3, 7-22.

—, 2015a, «Las dualidades secundarias de la escritura ibérica nororiental», ELEA 14, 305-357.

—, 2016, "Une inscription rupestre ibère inédite de Ger (Cerdagne) avec la formule neitin iunstir», Sources - Les cahiers de l'Âne Rouge 4, 13-28.

Ferrer, J. - Escrivà, V., 20I3, "Quatre noves inscripcions ibèriques pintades procedents de Llíria", $\mathrm{Pa}$ laeohispanica 13, 461-482.

Ferrer, J., Lorrio, A. J., VelazA, J. 2015, «Las inscripciones ibéricas en escritura suroriental del Castellar de Meca (Ayora)», Palaeohispanica 15, 161-176.

Gorrochategui, J., 1984, Onomástica indígena de Aquitania, Bilbao: UPV-EHU.

$M L H=$ J. Untermann, Monumenta Linguarum Hispanicarum, Wiesbanden, 1975-1997. 
Moncunill, N., 2007, Lèxic d'inscripcions ibèriques (1991-2006), Universitat de Barcelona, Tesis en xarxa TDX. http://diposit.ub.edu/dspace/bitstream/2445/41649/1/NMM_TESI.pdf

—, 2010, Els noms personals ibèrics en l'epigrafia antiga de Catalunya, Barcelona: IEC.

Orduña, E., 2005, «Sobre algunos posibles numerales en textos ibéricos», Palaeohispanica 5, 491-506.

—, 2006, Segmentación de textos ibéricos y distribución de los segmentos, Departamento de Filología Clásica. Facultad de Filología de la UNED, Madrid.

—, 2009, «De nuevo sobre el sufijo ibérico -te», Palaeohispanica 9, 501-514.

—, 2011, «Los numerales ibéricos y el protovasco», Veleia 28, 125-139.

Quintanilla, A., 2005, «Palabras de contenido verbal en ibérico», Palaeohispanica 5, 507-520.

Rodríguez Ramos, J., 2001, "El término (t)eban(en) en la lengua íbera: 'coeravit' vs. 'filius'», Arse 35, 59-85

—, 2005, "Introducció a l'estudi de les inscripcions ibèriques», Revista de la Fundació Privada Catalana per l'Arqueologia Ibèrica 1, 13-144.

—, 2014, «Nuevo Índice Crítico de formantes de compuestos de tipo onomástico íberos», Arqueoweb: Revista sobre Arqueología en Internet 15:1, 1-158.

Silgo, L., 2004, «Breves consideraciones sobre la piedra ibérica de Cruzy», Palaeohispanica 4, 195-197.

Simón, I., 2013, Los soportes de la epigrafía paleohispánica: inscripciones sobre piedra, bronce y cerámica, Zaragoza-Sevilla: Prensas universitarias de Zaragoza - Secretariado de Publicaciones Univ. Sevilla.

Solier Y., 1979, "Découverte d'inscriptions sur plombs en écriture ibérique dans un entrepôt de Pech Maho (Sigean)», Revue Archéologique de la Narbonnaise 12, 55-123.

Untermann, J., 1987, «La gramática de los plomos ibéricos», en: J. Gorrochategui - J.L. Melena - J. Santos (eds.), Studia Palaeohispanica. Actas del IV Coloquio sobre lenguas y culturas paleohispánicas (= Veleia 2-3), Vitoria, 35-56.

—, 1989, «Nova inscripció ibèrica sobre plom procedent del país dels ilergetes», Acta Numismàtica 19, 3944.

—, 1995, «La lengua ibérica. Nuestro conocimiento y tareas futuras», Veleia 12, 243-256.

—, 1999, «L'inscription sur pierre d'Ensérune, conservée dans le musée de Cruzy (Hérault)», Archéologie en Languedoc 23, 107-110.

—, 2002, «Dos nuevos textos ibéricos del sur de Francia», Palaeohispanica 2, 355-361.

—, 2002a, "Análisis e interpretación de la inscripción ibérica sobre piedra aparecida en la zona 18 del Puig de Sant Andreu (Ullastret)», Cypsela 14, 247-249.

—, 2005, "La lengua ibérica en el sur de Francia», en: Món Ibèric als Pä̈sos Catalans. XIII Col-loqui Internacional d'Arqueologia de Puigcerdà (14 i 15 de novembre de 2003). Homenatge a Josep Barberà i Farràs, Puigcerdà: Institut d'Estudis Ceretans, 1083-1100.

Valdeyron, J., 1998, «Pierre ibérique du Musée de Cruzy (F. 34)», Association Culturelle, Archéologique et Paléontologique du Cruzy, 1-4.

Velaza, J., 1991, Léxico de las inscripciones ibéricas (1976-1989), Barcelona: UB.

—, 1994, «Iberisch -eban, -teban», Zeitschrift für Papyrologie und Epigraphik 104, 142-150.

—, 2001, "Chronica epigraphica Iberica II: novedades y revisiones de epigrafía ibérica (1995-1999)", en: F. Villar - P. Fernández (eds.), Religión, lengua y cultura prerromanas de Hispania. Actas del VIII Coloquio sobre lenguas y culturas paleohispánicas, Salamanca, 639-662.

—, 2002, "Ibérico -te», Palaeohispanica 2, 271-275.

—, 2002a, "Et Palaeohispanica scripta manent: la epigrafía romana como modelo de las epigrafías paleohispánicas», Scripta manent. La memoria escrita de los romanos, Barcelona, 52-65.

—, 2004, «Eban, teban, diez años después», ELEA 5, 199-210.

—, 2013, «Tres inscripciones sobre plomo de La Carencia (Turís, Valencia)», Palaeohispanica 13, 539-550. 